Classification

Physics Abstracts

$07.80-61.70 \mathrm{~J}-62.20 \mathrm{~F}$

\title{
In situ deformation and description of mechanical behaviors in $\mathrm{Ni}_{3} \mathrm{Al}$
}

\author{
K. Jumonji and A. Sato \\ Department of Materials Science and Engineering, Tokyo Institute of Technology, 4259 Nagatsuta, \\ Midori-ku, Yokohama 227, Japan
}

(Received february 1, 1993; accepted march 18, 1993)

\begin{abstract}
In situ deformation has been conducted in the H-1250S HVEM with particular interest in finding correspondence between microscopic and macroscopic characteristics in strengthening of $\mathrm{Ni}_{3} \mathrm{Al}$. Among the various materials, $\mathrm{Fe}-\mathrm{Cr}-\mathrm{Ni}, \mathrm{Mo}, \mathrm{Ni}_{3} \mathrm{Al}, \mathrm{Fe}-\mathrm{Mn}-\mathrm{Si}, \mathrm{Cu}-\mathrm{Fe}$ and $\mathrm{Cu}-\mathrm{Ni}-\mathrm{Sn}$ examined in recent several years, discrepancy has been noted in the high temperature strengthening of $\mathrm{Ni}_{3} \mathrm{Al}$ in situ and bulk experiments. The serious structural differences found between the two types of experiments are compared and discussed in the light of the thin foil effect.
\end{abstract}

\section{Introduction.}

In situ deformation is a unique and powerful method in examining the basic properties of dislocation motion. A high voltage electron microscope is shown to be useful in examining dislocation motion in the state close to bulk samples (Saka et al., 1972, Saka and Imura, 1972, Tabata and Fujita, 1972, Fujita and Ueda, 1972, Mori and Fujita, 1977, Tabata et al., 1977, Martin and Kubin, 1979). Radiation damage may, however, ruin the original characteristics. Therefore, in some cases an electron microscope with a lower energy, say $200 \mathrm{kV}$, is more useful, as for example demonstrated in in situ experiments of $\mathrm{Ni}$ based $\mathrm{L1}_{2}$ ordered alloys (Caillard et al., 1988, Molenat and Caillard, 1991), Cu-Fe (Ishikawa and Sato, 1991) and Cu-Ni-Sn alloys (Sato et al., 1993).

In many cases in situ experiments have been conducted with particular interest in the basic properties. For example the main topics subjected in our recent studies are 1) Dislocation interaction with radiation induced defects (Suzuki et al., 1991, Suzuki et al., 1992), 2) Phase transformation governed by dislocation motion (Ishikawa and Sato, 1991), 3) Dislocation multiplication involved in $\gamma \rightarrow \varepsilon$ martensitic transformation (Hoshino et al., 1990), 4) Strengthening in a Cu-Ni-Sn alloy by spinodal decomposition (Sato et al., 1993), and 5) Strengthening in $\mathrm{Ni}_{3} \mathrm{Al}$ (Jumonji et al., 1993). The present paper will put major focus on the subject 5) with special interest in finding linkage between the in situ deformation of a thin foil specimen and the high temperature strengthening of a bulk specimen. 
At this point, we would like to introduce a few important observations made in the past in conjunction with the topics 1), 2), 3) and 4). In pursuing the above mentioned experiments, special techniques have been developed in order to overcome the difficulties encountered in the earlier studies.

In subject 1), use of baby cyclotron and irradiation of a jet polished specimen with the center thickness of $30 \mu \mathrm{m}$ was particularly advantageous in preparing a micro tensile specimen containing homogeneously distributed interstitial loops. In this in situ experiments the micro tensile specimen prepared without perforation was deformed in a HVEM by substantial amount cracking. This enabled us to study the dislocation interaction with different types of loops in bcc Mo (Suzuki $e t$ al., 1991) and in fcc Fe-Cr-Ni (Suzuki et al., 1992) single crystals.

In subject 2), $\gamma \rightarrow \alpha$ (bcc) martensitic transformation has been traced successfully by in situ experiments. The $\gamma \rightarrow \alpha$ transformation in a spherical Fe particle embedded in $\mathrm{Cu}$ matrix is found to be initiated by operation of double shears at the particle. It is also shown that once the transformation starts it completes spontaneously and the particle emits many dislocations explosively (Ishikawa and Sato, 1990). In this study, preparation of a micro tensile specimen without perforation was again key in introducing large deformation without cracking, since large deformation is necessary to induce $\gamma \rightarrow \alpha$ transformation into small Fe particles (Monzen, 1981).

In subject 3 ), the $\gamma \rightarrow \varepsilon$ transformation is shown to take place slowly under certain experimental condition by operation of a pole mechanism, which is found to satisfy a dislocation reaction

$$
(a / 2)[1 \overline{1} 0]+(a / 2)[011]+(a / 2)[0 \overline{1} 1] \rightarrow(2 a / 3)[1 \overline{1} 1]+(a / 6)[\overline{1} 12]
$$

where the perfect dislocations in the left hand side in the above equation form a subboundary.

Moving on to subject 4), we find the case that the microscopic dislocation structure is strictly related to the macroscopic strengthening despite that examination has been made on a thin foil specimen of $\sim 200 \mathrm{~nm}$ under a $200 \mathrm{kV}$ electron microscope. This is obviously due to the fact that origin of the hardening by spinodal decomposition is spaced sufficiently close $(\sim 10 \mathrm{~nm})$ compared with the specimen thickness $(\sim 200 \mathrm{~nm})$.

In comparison with the above mentioned subjects of 1), 2), 3) and 4), the anomalous strengthening of the $\mathrm{L}_{2}$ ordered alloy $\left(\mathrm{Ni}_{3} \mathrm{Al}\right)$, the subject 5) has gained considerable attention in recent years. But the strengthening mechanism has not necessarily been clarified to satisfaction. For one reason, a difficulty arises due to the fact that superdislocations move too fast to obtain direct evidence for the proposed strengthening mechanism at high temperatures (Nemoto et al., 1980). According to the recent in situ experiments by Caillard et al. (1988), and Molenat and Caillard (1992), the superdislocation motion is shown to occur in various processes involving (010) cross slip in atomistic scale. But it is not clear how these atomistic processes are related to the yielding of a bulk specimen. Apart from the detailed mechanisms discussed by many investigators in the past, it is the purpose of the present study to compare the stress required to move superdislocations in in situ and in bulk experiments, and to compare the dislocation structures developed by deformation in both cases.

\section{Experimental.}

A single crystal rod of $20 \mathrm{~mm}$ in diameter and $200 \mathrm{~mm}$ in length was grown by the usual Bridgeman method, vacuum sealed in a $\mathrm{SiO}_{2}$ tube and homogenized at $1473 \mathrm{~K}$ for 5 days. Composition of thus prepared single crystal was found to be $\mathrm{Ni}_{74.7} \mathrm{Al}_{25.3}$ according to chemical analysis. Crystallographic orientation and slip system geometry of the micro tensile specimen used in this study are shown in figure 1 . In situ deformations were made at $292 \mathrm{~K}$ and $690 \mathrm{~K}$ by applying tensile stress 
along the horizontal direction in figure 1 after giving prestrain by a few $\%$ in bulk by compression. Application of a prestrain to introduce homogeneously distributed immobile dislocations was helpful in suppressing local deformation in in situ experiments so that fresh dislocations could be introduced everywhere in the thin foil specimen. A tensile device used in a HVEM is shown in figure 2. This device is designed to make control of the stress level precise by use of a spring. With this device spontaneous deformation can be suppressed by lowering the stress by a small amount just after yielding. Detailed description of the tensile device has been given in the previous reports (Hoshino et al., 1990, Ishikawa and Sato, 1990, Suzuki et al., 1991, Suzuki et al., 1992, Jumonji et al., 1993).
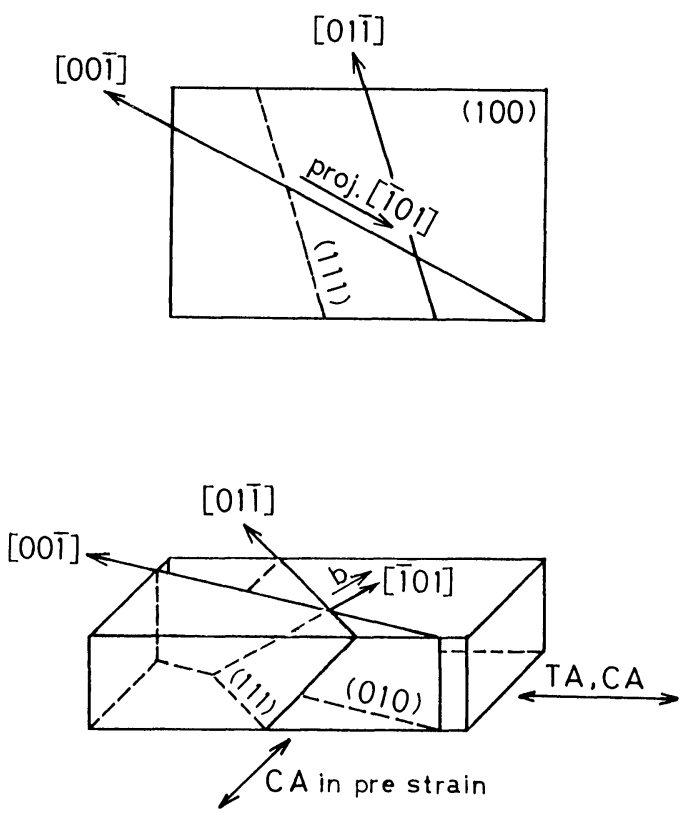

Fig. 1. - Slip system geometry of a micro tensile specimen and a bulk specimen used in the present study. Note that tensile axis in our in situ deformations and compression axis in the bulk deformation are taken along the same direction. The upper figure shows top view of a micro tensile specimen.

The bulk experiments were made by compression by using a rectangular specimen of $2 \times 5 \times$ $5 \mathrm{~mm}^{3}$ orientated as shown in figure 1 . The electron microscopic observations after bulk deformation were made by slicing the deformed sample along the primary slip plane and stopping the electropolishing just before perforation. In this manner dislocation rearrangement occasionally induced by thin foil preparation could be avoided effectively.

\section{Experimental results and discussions.}

3.1 Deformation AT ROOM TEMPERATURE. - Figure 3 compares the dislocation structures developed by in situ deformation and by bulk deformation at room temperature. Dislocations introduced by in situ deformation are numbered in the order of the appearance in the micrographs (a) and (b) taken at different stress levels by operating $\mathrm{g}=00 \overline{2}$ for which the primary dislocations appear with strong contrast. The dislocations introduced by prestrain in compression are not visible with this $\mathbf{g}$. It was confirmed by operating other $\mathbf{g}$ that the dislocations introduced by the 


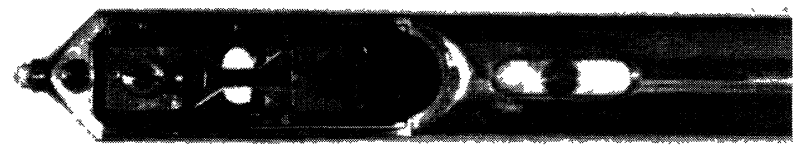

(a)

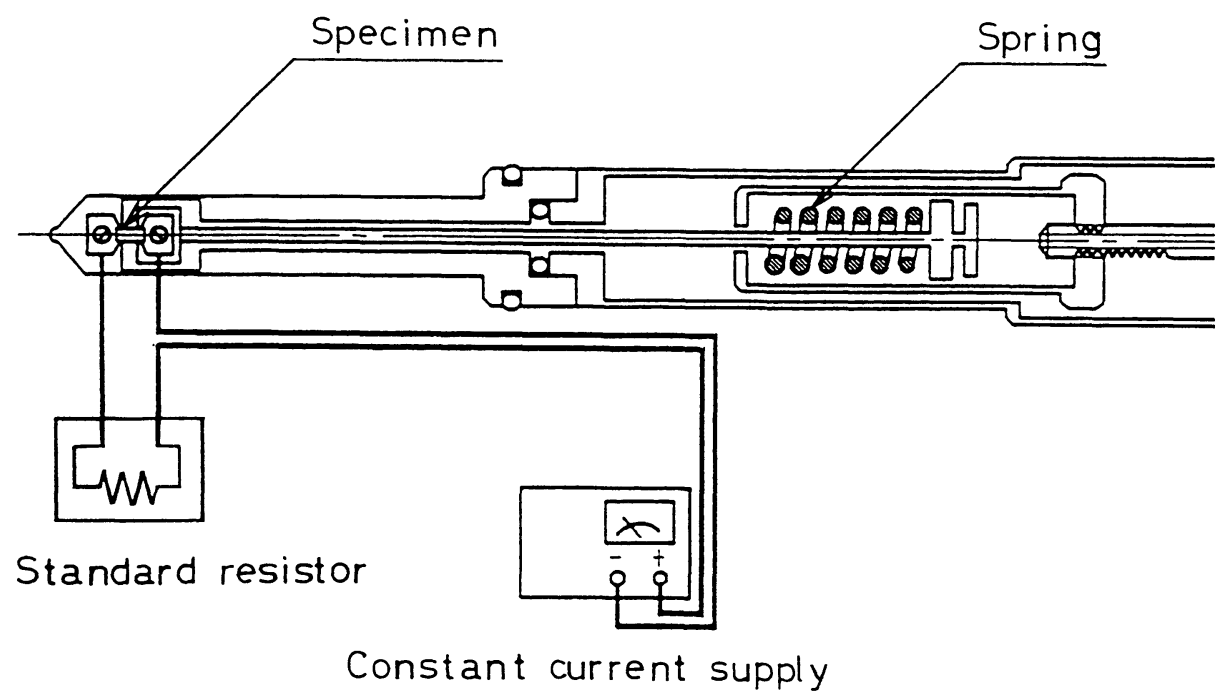

(b)

Fig. 2. - A tensile test device used in a HVEM (a) and the schematic drawing (b). The specimen was heated by a DC current and the temperature was measured and controlled by use of a standard resistor connected in series.

compression did not move in the in situ deformation. Dislocations 1 and 2 were introduced at a shear stress of $40 \mathrm{MPa}$ and 3 7 at 67 68 MPa. Before taking micrographs at each deformation step, the load was lowered by a few \% in order to stop the dislocation motion and to detect the structural change in fine scale. Note that a curved dislocation 1 has moved out of the sight by increasing the stress up to $68 \mathrm{MPa}$. Slip traces were also formed along the (111) trace at this stress level in figure $3 \mathrm{~b}$, suggesting that many primary dislocations passed through the viewing sight in addition to the dislocations $5 \sim 7$ stopped along the trace. It is, however, not known to what stage this dislocation structure would correspond on a macroscopic stress-strain curve.

In the present in situ deformation, although the dislocations in motion could not be detected dynamically, it was confirmed that some dislocations once stopped, such as 1 and 2, moved again 

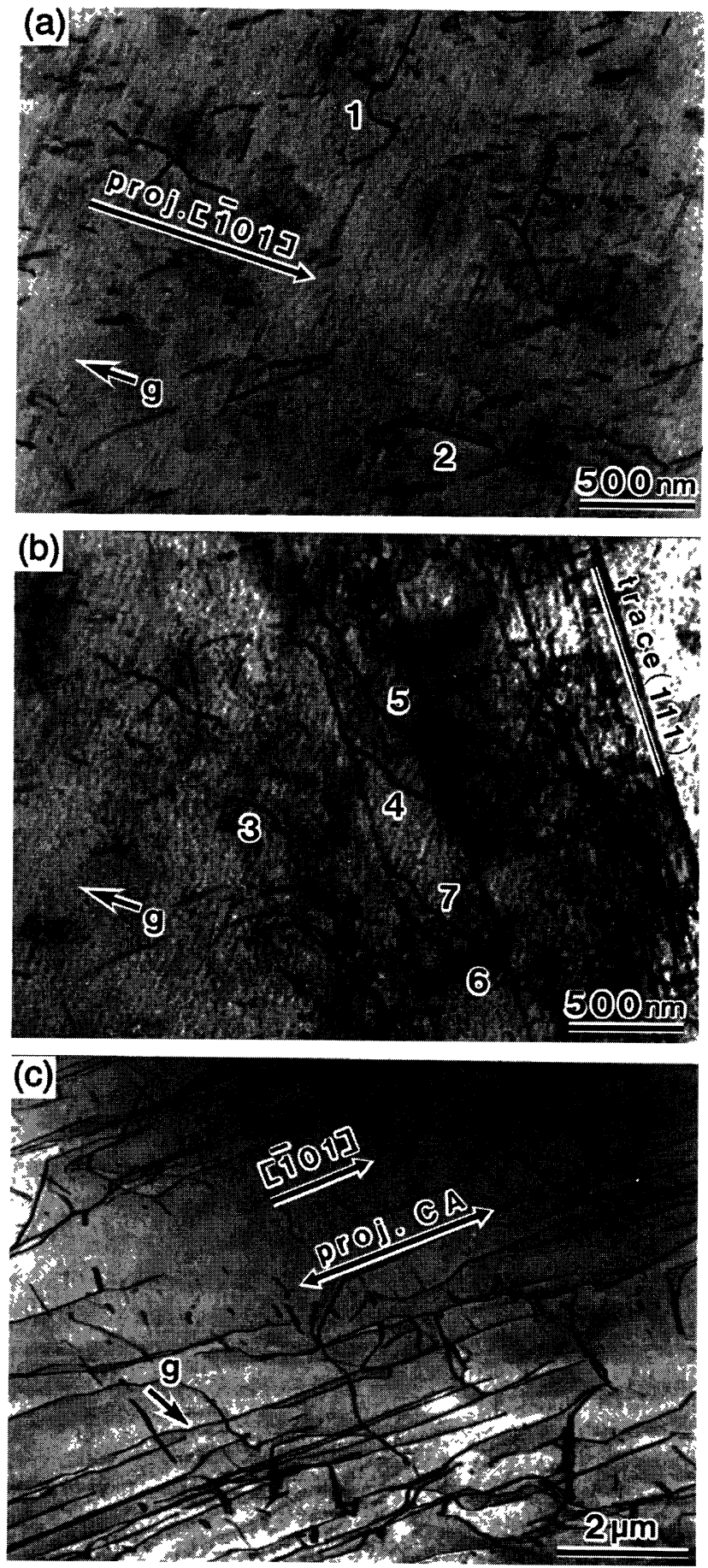

Fig. 3. - Comparison of dislocation structures developed at room temperature by in situ (a), (b) and bulk (c) deformations. Stressed up to $40 \mathrm{MPa}$ in (a) and $68 \mathrm{MPa}$ in (b) in the resolved shear stress for [101] (111). The operating $\mathbf{g}$ is $00 \overline{2}$ for (a) and (b). (c) A thin foil specimen sliced along the primary slip plane after compression by $1.2 \%$ in bulk. The operating $\mathrm{g}$ is $\overline{2} 20$. 
at a higher stress level. Though we didn't show photographs here, the dislocation 1 which had less screw segments moved prior to the motion of the dislocation 2 at the lower stress level. The dislocation 2 which had the longer screw segment moved after substantial deformation as evidenced in the generation of clear (111) slip trace. Judging from the coincidence of the locations of the dislocations 2 and 6, it is speculated that the dislocation 2 has become mobile under the influence of a strong stress field generated by the pile-up dislocations such as 6,7 and possibly other dislocations which has passed away.

The present observation at room temperature supports a recent model of superkink motion reported by Hirsch (1992) in a sense that the thermally activated overcoming of the locking shall be assisted by a long range stress. The thermal stress component is therefore small but not zero at room temperature. Detailed discussion on this point will be presented elsewhere with the strain rate sensitivity data (Jumonji et al., 1993). The strengthening at a higher temperature but below the peak temperature is, however, controlled totally in athermal manner as also discussed (Jumonji et al., 1993).

A dislocation structure developed in bulk deformation at room temperature is shown for comparison in figure $3(\mathrm{c})$. The deformation was given to $1.2 \%$ by compression. The foil specimen was prepared by slicing the deformed sample along the primary slip plane (111) and thinning it down to $\sim 1 \mu$ m without perforation. The dislocation configuration found in figure 3 (c) representing a bulk structure, is noteworthy and is quite different from that developed in the in situ deformation shown in (a) and (b). The structure in (c) is characterized by the homogeneously distributed dislocations with preference in screw orientation. Suppose that non-screw segments linked to the immobile screw dislocations move in a bulk specimen, the stress required for the motion of the non-screw segments should be given by an Orowan type of stress. It is estimated to be $50 \mathrm{MPa}$ in good accordance with the flow stress of $60 \mathrm{MPa}$ in resolved shear stress at $1.2 \%$ strain in a bulk test. But this value is somewhat larger than $40 \mathrm{MPa}$ at which fresh dislocations were introduced in the in situ experiment. The stress may be amplified near the center of a dip in the jet polished specimen since the amplification factor is known to be approximately 1.2 in the present specimen without perforation, according to the previous studies (Hoshino et al., 1990, Suzuki et al., 1991, Suzuki et al., 1992).

3.2 Deformation AT $500 \mathrm{~K}$ AND $690 \mathrm{~K}$. - At a higher temperature, the discrepancy in the flow stress as well as in the dislocation structure becomes more pronounced between bulk and in situ experiments. Figure 4 compares the dislocation structures developed in bulk and in situ experiments at higher temperatures. Figure $4 \mathrm{a}$ shows the structure obtained by deformation at $68 \mathrm{MPa}$ at room temperature corresponding to figure $3 \mathrm{~b}$. When this specimen was heated up to $690 \mathrm{~K}$ and strained at this temperature, deformation started at $78 \mathrm{MPa}$ and many fresh dislocations were introduced as shown in figure $4 \mathrm{~b}$. In the figure $S$ identifies a stable reference dislocation remained without motion by the high temperature deformation. The stress required for a dislocation to move at $690 \mathrm{~K}$ is, thus, found to be not much larger than the value $68 \mathrm{MPa}$ obtained at room temperature and is substantially smaller than the yield stress found in bulk deformation at high temperatures. For instance, the present single crystal yields at $200 \mathrm{MPa}$ in resolved shear stress at $500 \mathrm{~K}$.

The origin of the large discrepancy in the yield stress can be seen by comparison of figure $4 \mathrm{~b}$ and figures $4 \mathrm{c}$ and $\mathrm{d}$, showing the dislocation structure obtained after deformation at $500 \mathrm{~K}$ by $1.0 \%$ in compression. Primary dislocations on the (111) slip planes are visible in strong contrast but the secondary dislocations on the (111) planes are not visible in (c) and vice versa for (d). Complete disappearance of either secondary or primary dislocations in (c) and (d) means that these dislocations are superdislocations with the Burgers vector of a $\langle 110\rangle$ type. It is interesting to note that separation between a pair of screw dislocations linked to non-screw segment gives the 

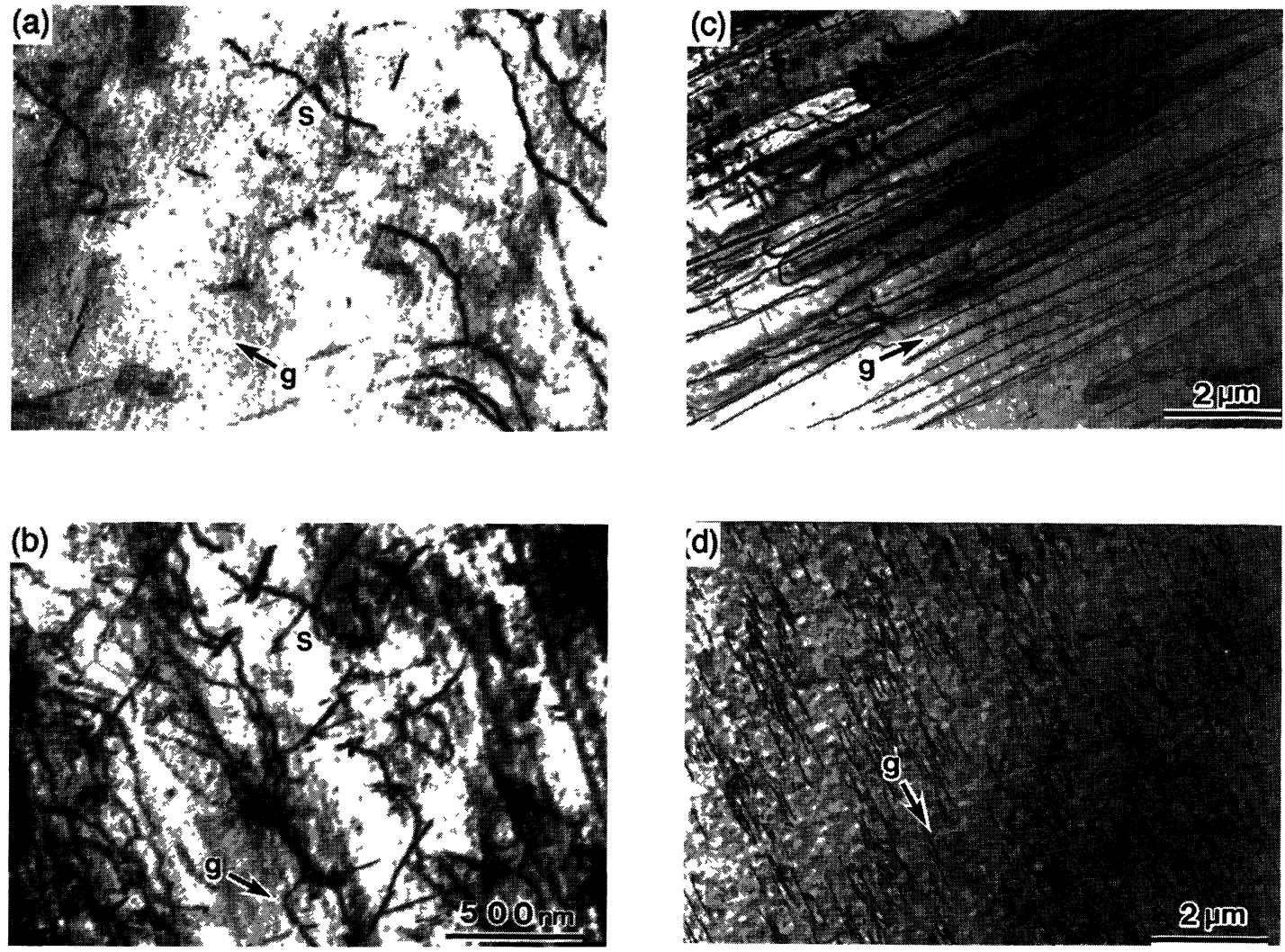

Fig. 4. - Comparison of the dislocation structures developed at high temperatures by in situ (b) and bulk (c), (d) deformations. (a) After deformation at room temperature corresponding to Fig. 3b. (b) After deformation at $78 \mathrm{MPa}$ at $690 \mathrm{~K}$. The operating $\mathrm{g}$ are $00 \overline{2}$ for (a) and $002 \mathrm{for}$ (b). (c) and (d) show the same area of a thin foil specimen sliced along the primary slip plane after deformation at $500 \mathrm{~K}$ by $1.0 \%$ in compression. The operating $g$ are $\overline{2} 02$ for (c) and $11 \overline{1}$ for (d).

Orowan type of stress of $180 \mathrm{MPa}$ in good accordance with the aforementioned macroscopic flow stress of $200 \mathrm{MPa}$. In contrast, a small value of $78 \mathrm{MPa}$ was obtained in the in situ deformation at $690 \mathrm{~K}$. This discrepancy may be attributed to a surface effect arising in in situ experiments. The stress which is required to move the non-screw dislocations linked to short screw segments should not be greater than half of the Orowan type of yield stress because the effective pinning distance will increase at least by a factor of two due to the presence of the stress free surfaces. In other words, the deformation in the in situ experiment proceeding without production of screw dislocation segments would not provide correct information in dealing with the strengthening mechanism in bulk specimens at high temperatures.

The dislocation structures on the primary and secondary slip planes are similar as found in the stereo pair of micrographs shown in figure 5. From the three dimensional view of the dislocation structure, it is confirmed that long and straight screw dislocations as well as the non-screw segments linked to them lie totally on the flat (111) planes at least within the scale of strain contrasts. Similarly the straight secondary dislocations with the Burgers vector $a[101]$ penetrating through the foil specimen lie totally on the $(\overline{111})$ planes inclined with respect to the (111) planes by $71^{\circ}$. The total lengths are also same for the set of dislocations lying on each type of slip planes. Re- 


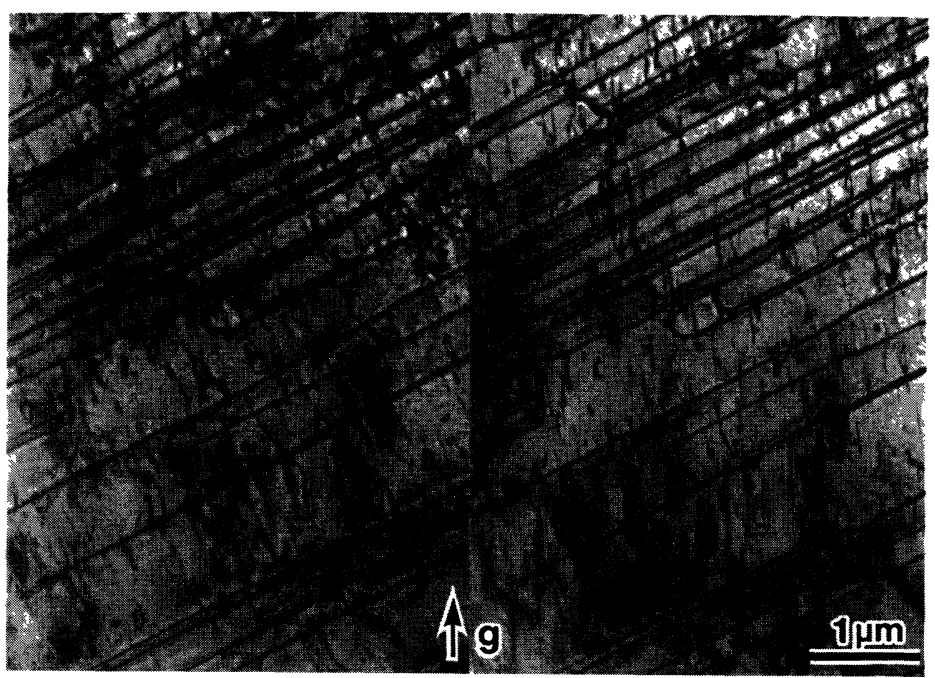

Fig. 5. - Stereoscopic observation of a dislocation structure developed by $4.2 \%$ compression at $500 \mathrm{~K}$. A thin foil specimen sliced along the primary slip plane is viewed along two directions rotated by $15^{\circ}$ around $\mathrm{g}=0 \overline{2} 2$.

minding that the Schmid factor is not much different for the primary $(0.50)$ and secondary $(0.46)$ slip systems and that the flow curve is characterized by a large work hardening, the above observation can be explained easily by considering motion of non-screw dislocation linked to immobile straight screw dislocations.

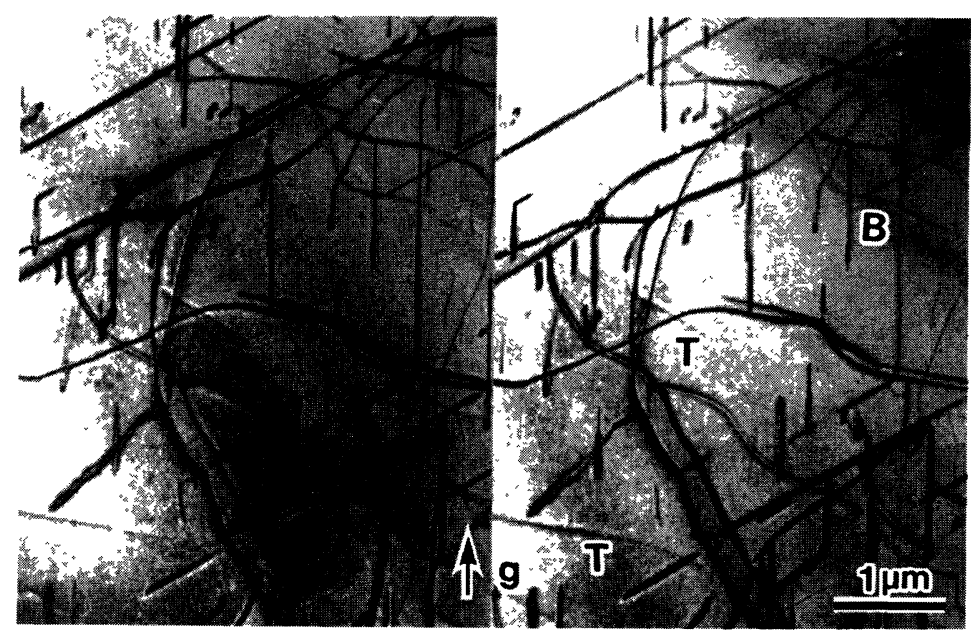

Fig. 7. - Stereoscopic observation of a dislocation structure in the area shown in figure 6. the photographing conditions are same as figure 5. Note that faint contrasts are located at top (T) or bottom (B) of the foil surface. 



Fig. 6. - A dislocation structure developed by $5.2 \%$ compression at $77 \mathrm{~K}$. Observations of a thin foil specimen sliced along the primary slip plane. The operating $\mathbf{g}$ are $\overline{2} 02$ for (a), $20 \overline{2}$ for (b) and $\overline{1} 1 \overline{1}$ for (c). 
3.3 DEFORMATION AT $77 \mathrm{~K}$. - In contrast to the rather simple dislocation structure but wit high density of screw dislocations introduced at $500 \mathrm{~K}$ (Fig. 4), the dislocation structure developer by bulk deformation at $77 \mathrm{~K}$ is complicated as shown in figure 6 . Burgers vectors were analyzed by taking 12 micrographs under different two beam conditions with $\mathbf{g}= \pm \overline{1} 11, \pm 1 \overline{1} 1, \pm 11 \overline{1}, \pm 20 \overline{2}$, $\pm 02 \overline{2}$, and $\pm 2 \overline{2} 0$ among which the three representative micrographs are shown in figure 6 . Disappearance of the gently curved dislocations $\mathrm{A}$ in (c) for $\mathrm{g}=\overline{1} 1 \overline{1}$ indicates that these dislocations are the primary dislocations with the Burgers vector $a[10 \overline{1}]$ lying on (111) planes. The strain contrasts remaining in figure $6 \mathrm{c}$ also lie on (111) primary slip planes and terminate inside the foil as found in the stereo pair of micrographs shown in figure 7 . These contrasts did not disappear in any of the 12 different $\mathrm{g}$ listed above. From the width change of the segmented dislocations upon reversal of $\mathbf{g}$ in (a) and (b), these segments, terminating inside the foil, are revealed to have the characteristics of dipole dislocations. Although detailed structure of the segments are not determined at present, it is apparent that the straight segments aligned along the $\pm[10 \overline{1}]$ and $\pm[01 \overline{1}]$ directions were generated by motion of the superdislocations possibly with jogs such as marked by $J$ in figure 6 . The curved dipole segments in figure 6 are aligned neither along the $\pm[10 \overline{1}]$ nor along the $[01 \overline{1}]$ directions. Judging from the zigzag configuration of these dislocations, the curved segments may also result from the dislocation motion with dipole dragging along the combined direction of $\pm[10 \overline{1}]$ and $\pm[01 \overline{1}]$. Several faint contrasts in figure 7 are another unique structure found after deformation at $77 \mathrm{~K}$. The fact that these contrasts are located at top (T) or bottom (B) surfaces of the foil as found in the stereo microscopy implies that they may have undergone rearrangements during specimen preparation.

It is important to note here that the straight dislocations formed along the $\pm[10 \overline{1}]$ direction were introduced by entirely different mechanisms at $77 \mathrm{~K}$ and at $500 \mathrm{~K}$. Jog hardening plays an important role at low temperatures in absence of point defect diffusion while locking of screw dislocations by Kear-Wilsdorf mechanism becomes predominant at high temperatures. The complete locking without unpinning will lead to Orowan type of athermal strengthening in a bulk specimen. The another characteristic difference resulting from the transient in the strengthening mechanism is the dislocation density. It is much higher after deformation at a higher temperature as found in comparison of figures $3 \mathrm{c}, 4 \mathrm{c}$, $\mathrm{d}$ and 6 , obtained after straining by $1.2 \%, 1.0 \%$ and $5.2 \%$, respectively. It has also become evident in these micrographs that deformation proceeds mainly by single slip up to $5.2 \%$ in compression at $77 \mathrm{~K}$ in the present specimen while substantial secondary slip has taken place at $1.0 \%$ strain at $500 \mathrm{~K}$. From the present observations, it is suggested that the rapid work hardening due to $\mathrm{KW}$ locking plays the essential role in the high temperature srengthening through the change in the total dislocation configuration in a bulk specimen. A rather small strengthening observed at $690 \mathrm{~K}$ in the in situ deformation may thus be attributed to the thin foil effect in the present study.

\section{Conclusions.}

Dislocation motion in a $\mathrm{Ni}_{3} \mathrm{Al}$ single crystal has been examined at $292 \mathrm{~K}$ and $690 \mathrm{~K}$ by in situ deformations in a HVEM. Microstructures in $\mathrm{Ni}_{3} \mathrm{Al}$ introduced by bulk deformations at $77 \mathrm{~K}$, $292 \mathrm{~K}$ and 500 has also been examined with special caution in preserving it without dislocation rearrangement during thin foil preparation. It has become evident that the dislocation motion confined to a narrow slip plane in the in situ deformation takes place in a different manner from a bulk specimen. The high temperature strengthening in a bulk specimen is thus controlled by the total dislocation configuration caused by $\mathrm{KW}$ locking in athermal manner but not strictly by simple locking-unlocking events. 


\section{References}

Caillard D. , Clement N., Couret A., Lours P. and Coujou A., Phil. Mag. Lett. 58 (1988) 263.

FujITA H. and UedA S., Acta Met. 20 (1972) 759.

HiRSCH P.B., Phil. Mag. 65 (1992) 569.

Hoshino Y., NAKAMURA S., IshIKAWA N. and SaTo A., Mater. Sci. Forum (ICOMAT-89), 56-58 (1990) 643.

IsHikawa N. and SATo A., Phil. Mag. A61 (1990) 929.

Ishikawa N. and Sato A., Phil. Mag. A64 (1991) 387.

Jumonji K., Shinohara T., Kato M. and Sato A., Phil. Mag. A67 (1993) 627.

Jumonji K., Ueta S., Kato M. and Sato A., Phil. Mag. (1993) submitted.

MARTIN J.L. and KUBIN L.P., phys. stat. sol. (a) 56 (1979) 487.

Molenat G. and Caillard D., Phil. Mag. A64 (1991) 1291.

Molenat G. and Caillard D., Phil. Mag. A65 (1992) 1327.

MORI T. and FUJITA H., Trans. JIM 18 (1977) 17.

MONZEN R., SATO A. and MORI T., Trans. JIM 22 (1981) 65.

NEMOTO M., HondA T., ECHIGOYA J. and SuTo H., J. Japan Inst. Metals 44 (1980) 933.

SAKa H., Noda K. and ImURA T., Japan J. Appl. Phys. 11 (1972) 1357.

SAKA H. and ImURA T., J. Phys. Soc. Japan 32 (1972) 702.

Sato A., Tamura K., Ito M., Kato M. and Mori T., Acta Met. 41 (1993) 1047.

Suzuki M., Fujimura A., Sato A., Nagakawa J., Yamamoto N. and Shiraishi H., Phil. Mag., A64 (1991) 395.

Suzuki M., Sato A.,Mori T., Nagakawa J., Yamamoto N. and Shiraishi H., Phil. Mag., A65 (1992) 1309. Tabata T. and Fujita H., J. Phys. Soc. Japan 32 (1972) 1536.

Tabata T., Nakajima Y. and Fujita H., Japan J. Appl. Phys. 16 (1977) 2011. 